\title{
A Condensed, Scalable Synthesis of Racemic Koningic Acid
}

Guillaume Barbe, ${ }^{1}$ David Chai, ${ }^{2}$ Bin Chen, ${ }^{2}$ Daniel Guay, ${ }^{2}$ Eric Levesque, ${ }^{2}$ John Mancuso, ${ }^{2}$ and Brian DeChristopher ${ }^{*}$

1. Rheos Medicines Inc. 38 Sidney St. Suite 200, Cambridge, MA 02139

2. NuChem Therapeutics Inc. 2350 Cohen, Suite 201, Saint-Laurent, QC Canada, H4R 2N6

KEYWORDS Koningic acid, heptelidic acid, glycolysis inhibitor, total synthesis

bdechristopher@rheosrx.com

\section{Supporting Information}

${ }^{1} \mathrm{H}$ NMR spectrum of (iodomethyl)triphenylphosphonium iodide (400 MHz, DMSO-d6) ........ S2

${ }^{1} \mathrm{H}$ NMR spectrum of 2,2-dimethyl-1,3-dioxan-5-one $\left(400 \mathrm{MHz}, \mathrm{CDCl}_{3}\right)$............................. S3

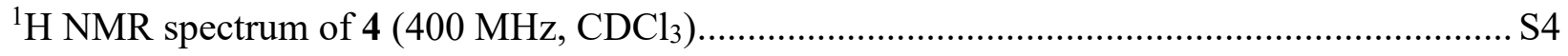

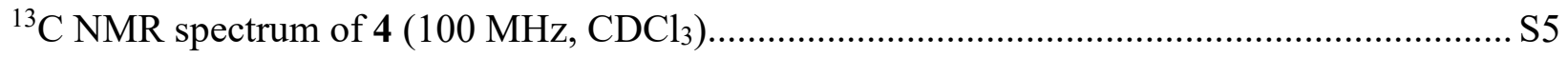

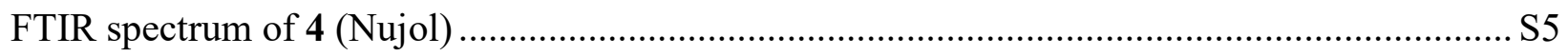

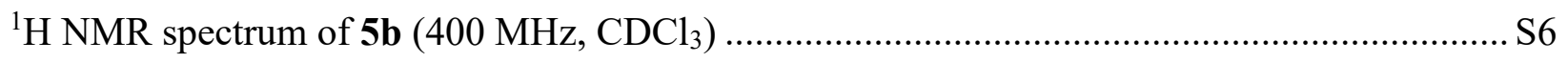

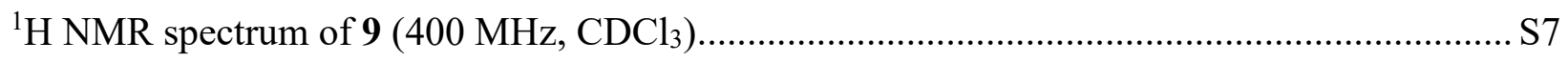

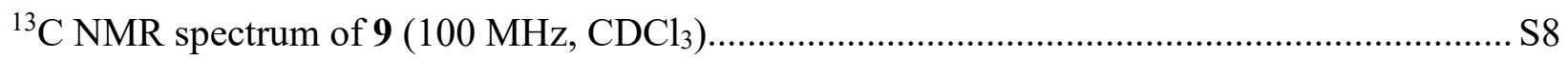

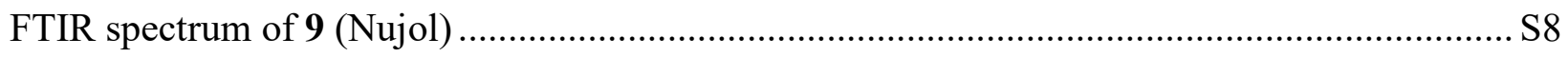

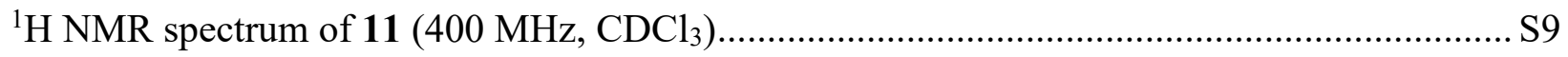

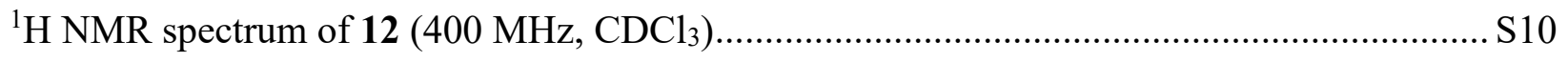

${ }^{1} \mathrm{H}$ NMR spectrum of 13 (400 $\left.\mathrm{MHz}, \mathrm{CDCl}_{3}\right)$................................................................. S11

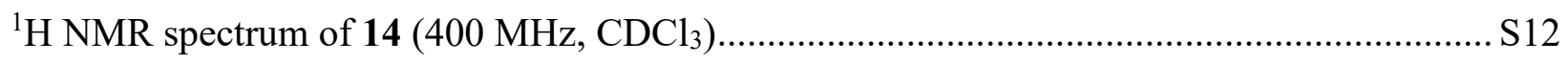

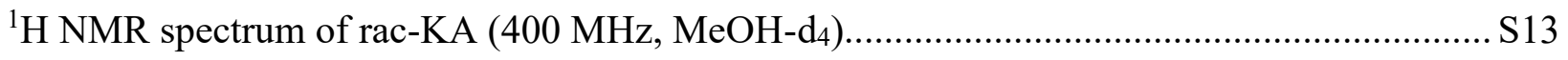


${ }^{1} \mathrm{H}$ NMR spectrum of (iodomethyl)triphenylphosphonium iodide (400 MHz, DMSO-d6)

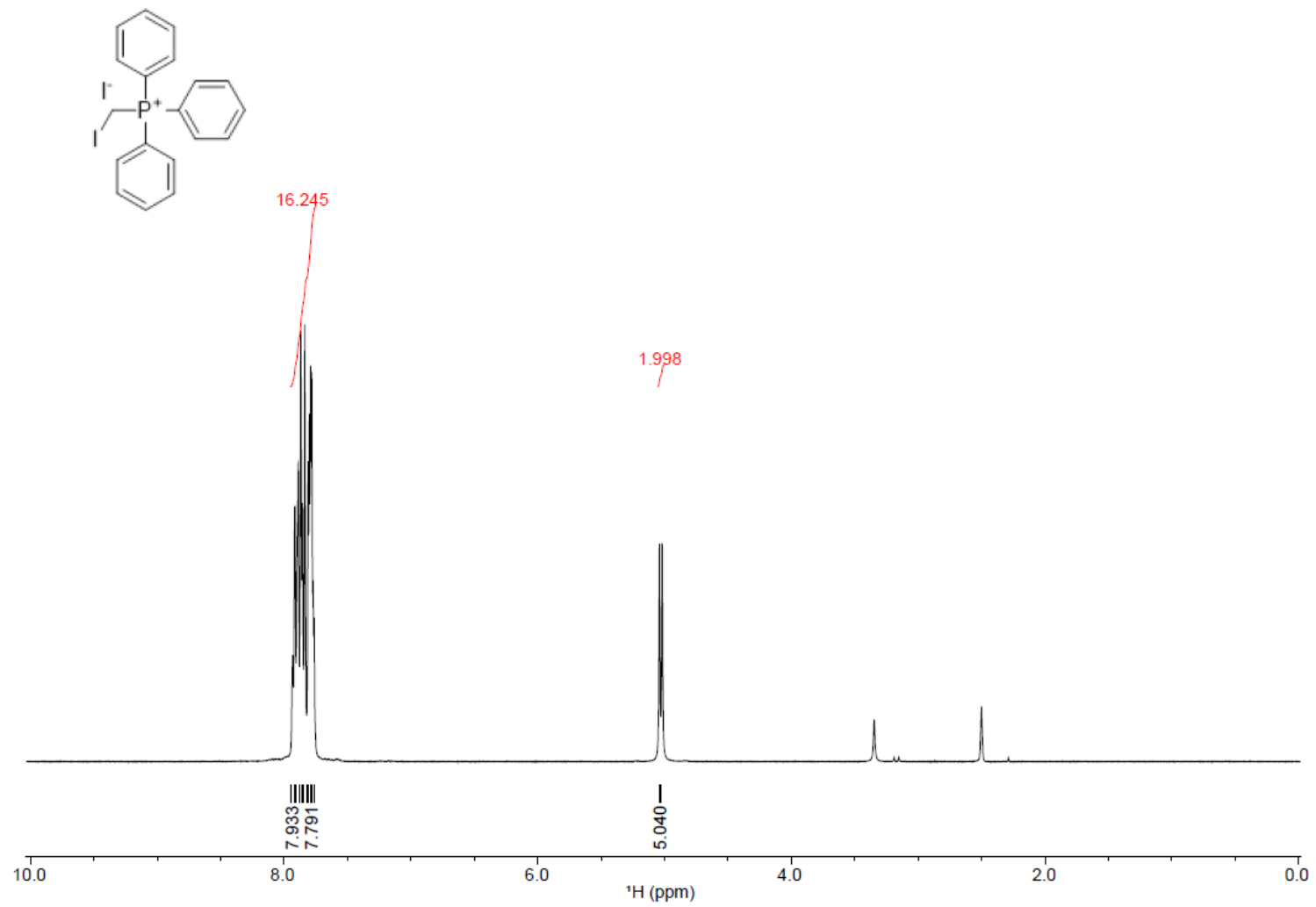


${ }^{1} \mathrm{H} N M R$ spectrum of 2,2-dimethyl-1,3-dioxan-5-one (400 $\mathrm{MHz}, \mathrm{CDCl}$ )

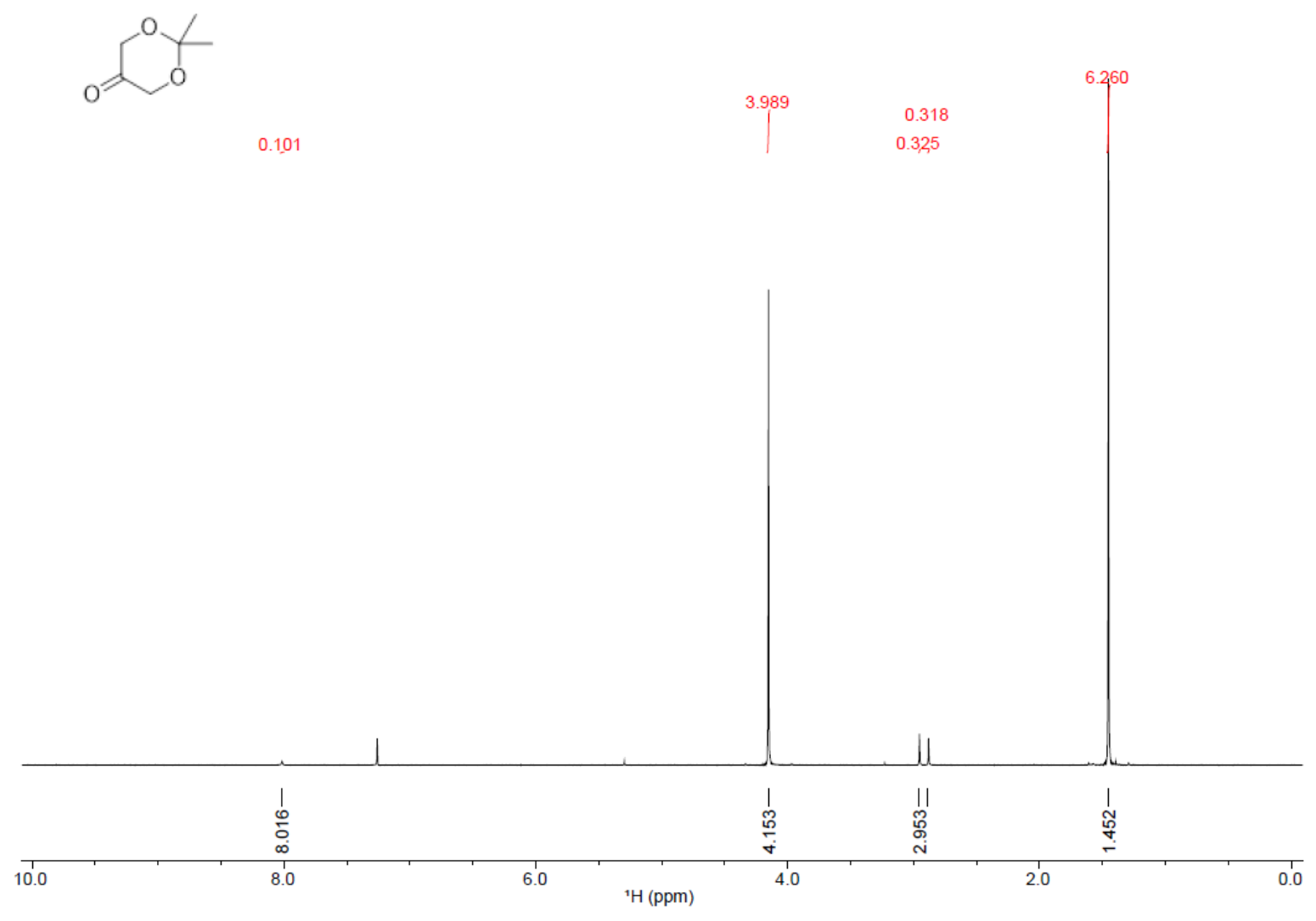


${ }^{1} \mathrm{HNMR}$ spectrum of $4\left(400 \mathrm{MHz}, \mathrm{CDCl}_{3}\right)$

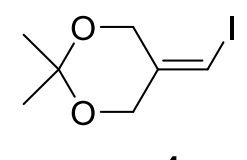

4

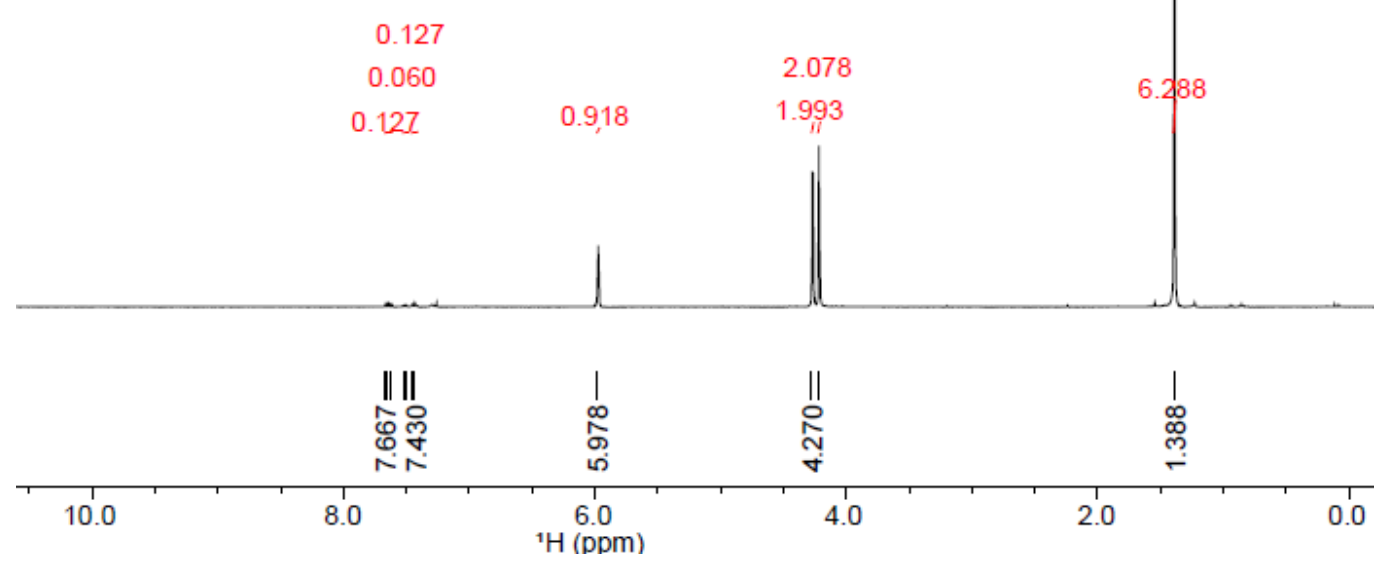


${ }^{13} \mathrm{C} \mathrm{NMR}$ spectrum of $4\left(100 \mathrm{MHz}, \mathrm{CDCl}_{3}\right)$<smiles>CC1(C)OCC(=CI)CO1</smiles>

4

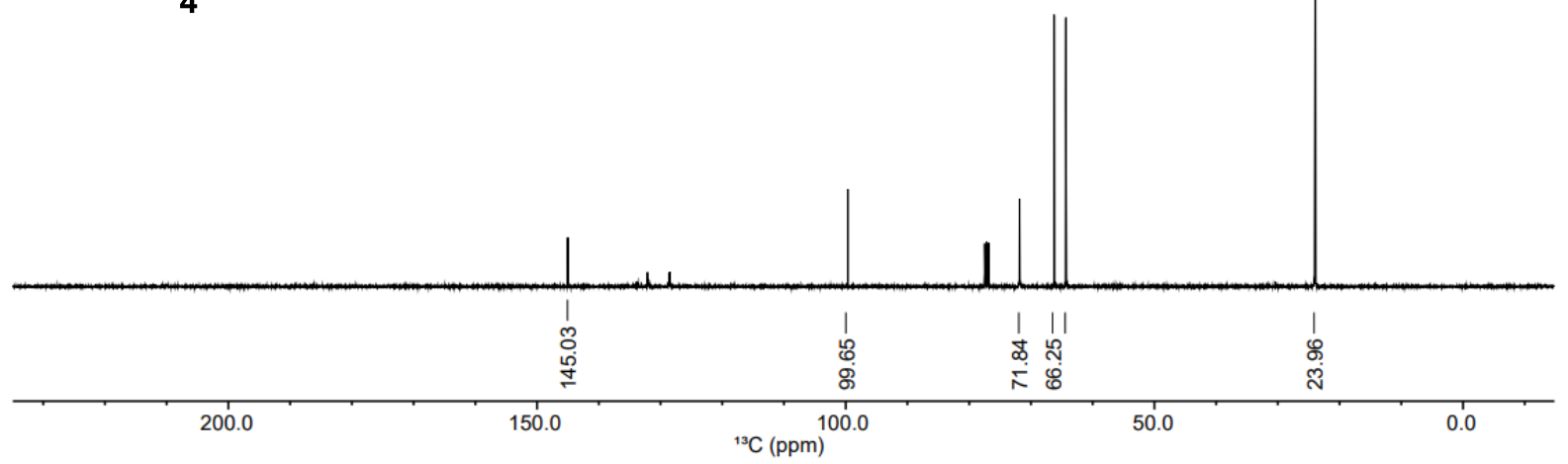

FTIR spectrum of 4 (Nujol)

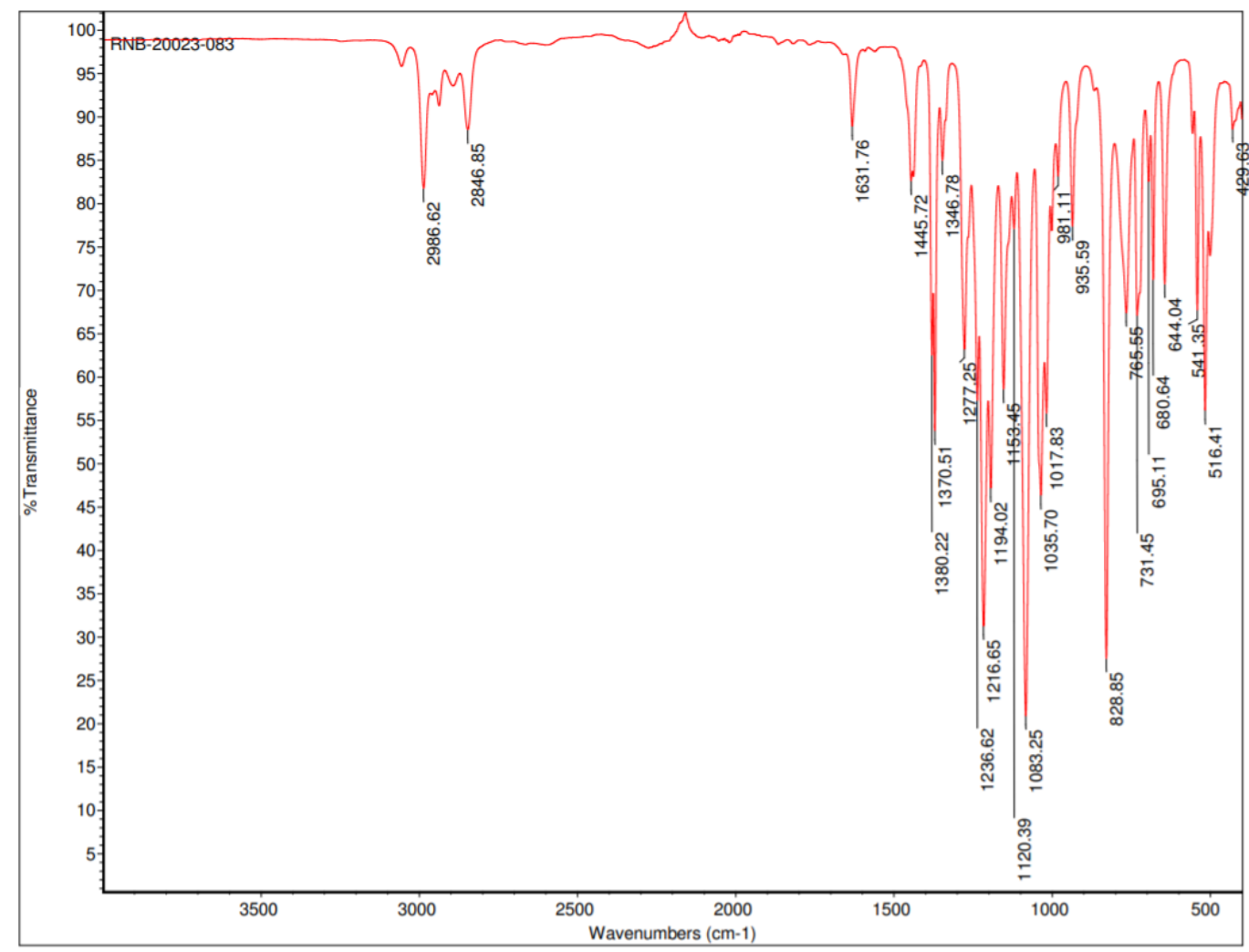


${ }^{1} \mathrm{H} N M R$ spectrum of $\mathbf{5 b}\left(400 \mathrm{MHz}, \mathrm{CDCl}_{3}\right)$
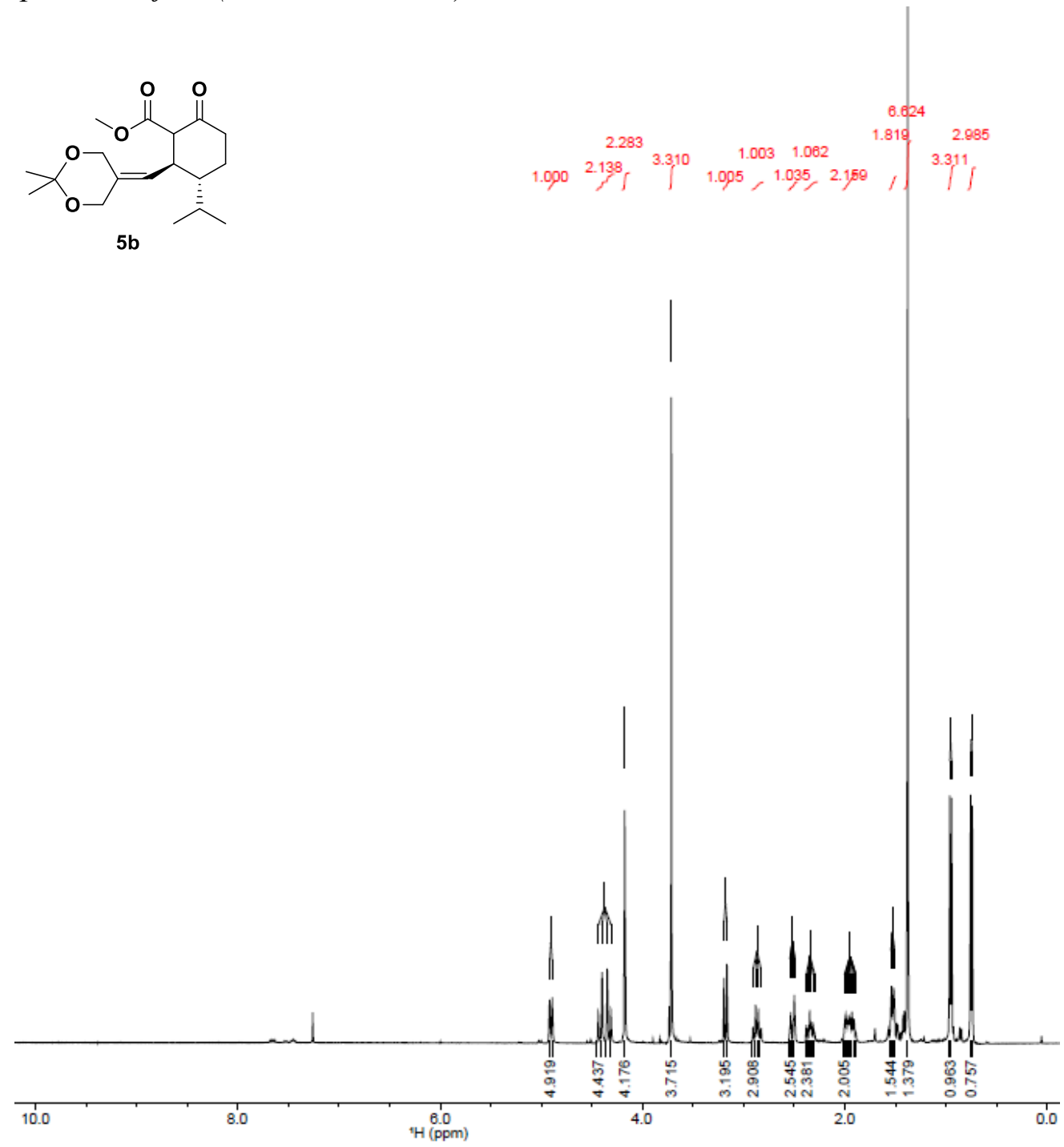
${ }^{1} H$ NMR spectrum of $9\left(400 \mathrm{MHz}, \mathrm{CDCl}_{3}\right)$
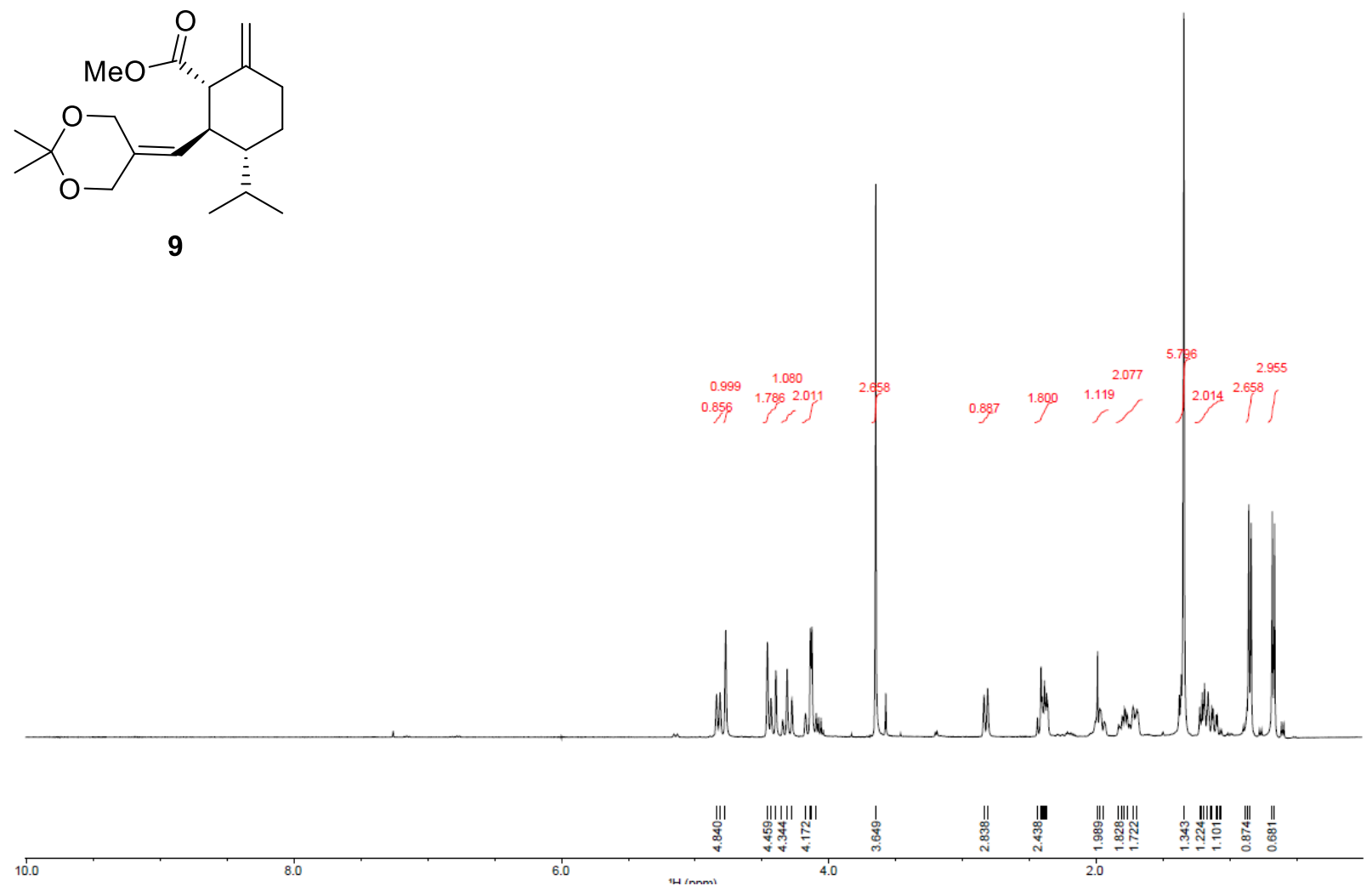
${ }^{13} \mathrm{C} \mathrm{NMR}$ spectrum of $9\left(100 \mathrm{MHz}, \mathrm{CDCl}_{3}\right)$<smiles>C=C1CC[C@H](C(C)C)[C@H](C=C2COC(C)(C)OC2)C1C(=O)OC</smiles>

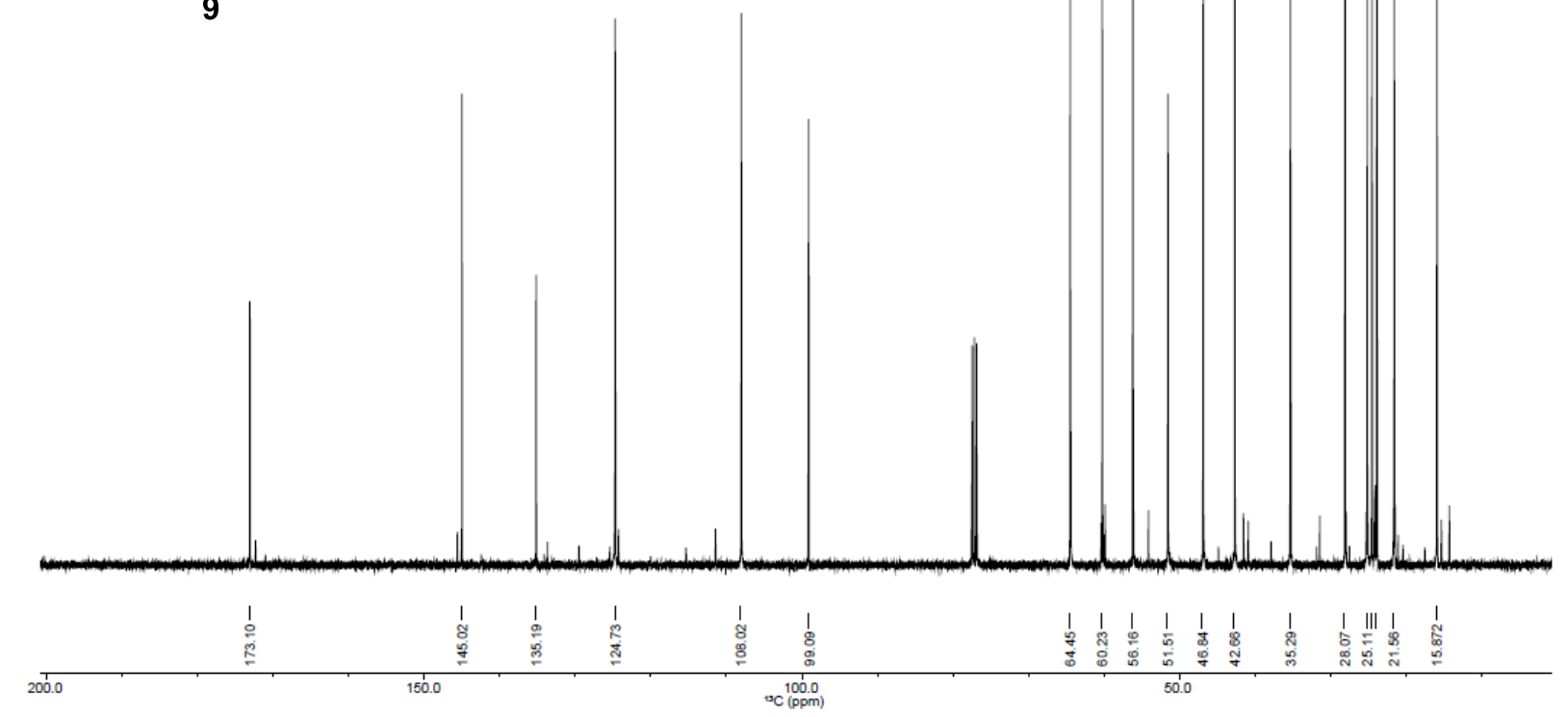

\section{FTIR spectrum of 9 (Nujol)}

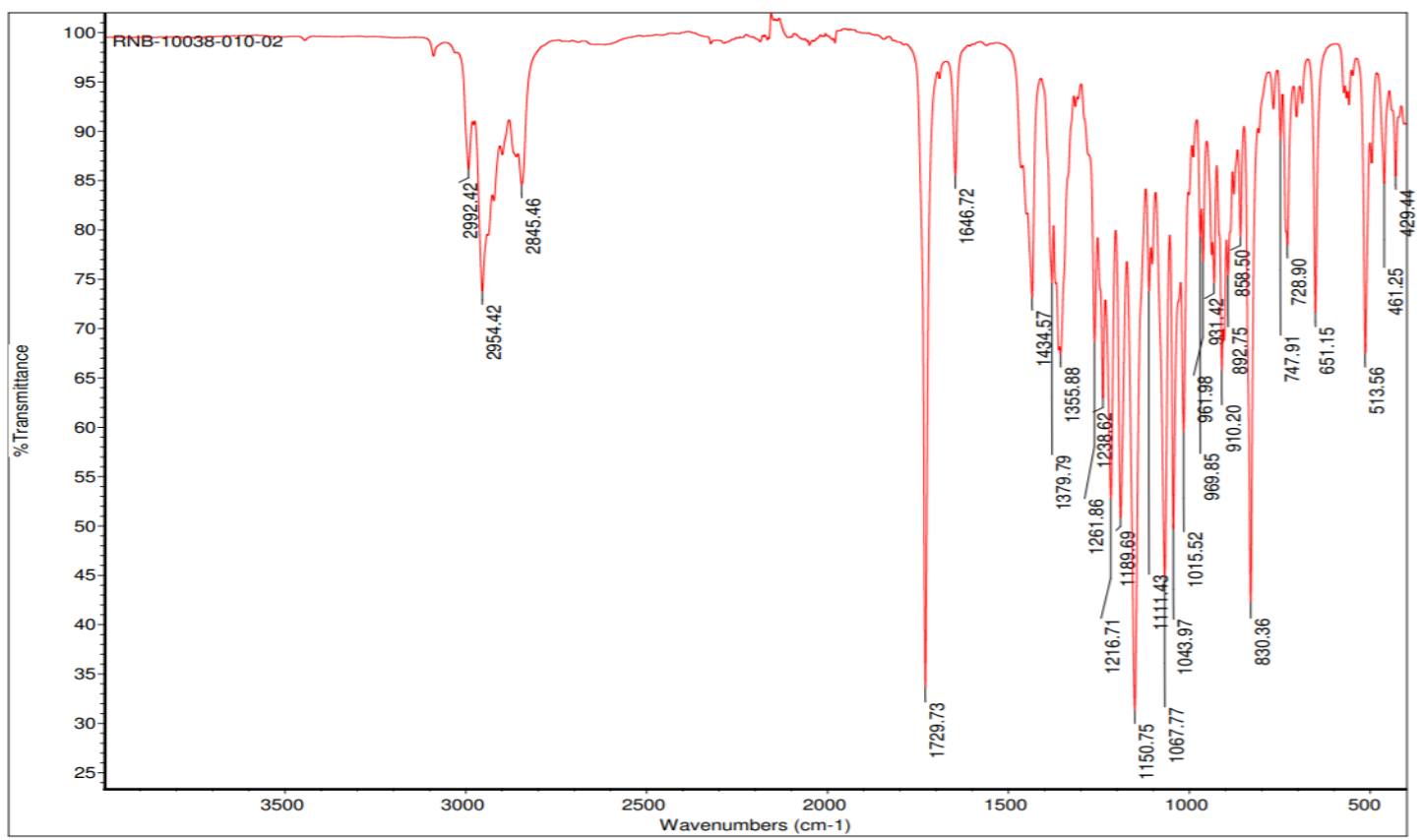


${ }^{1} \mathrm{H} N M R$ spectrum of $11\left(400 \mathrm{MHz}, \mathrm{CDCl}_{3}\right)$

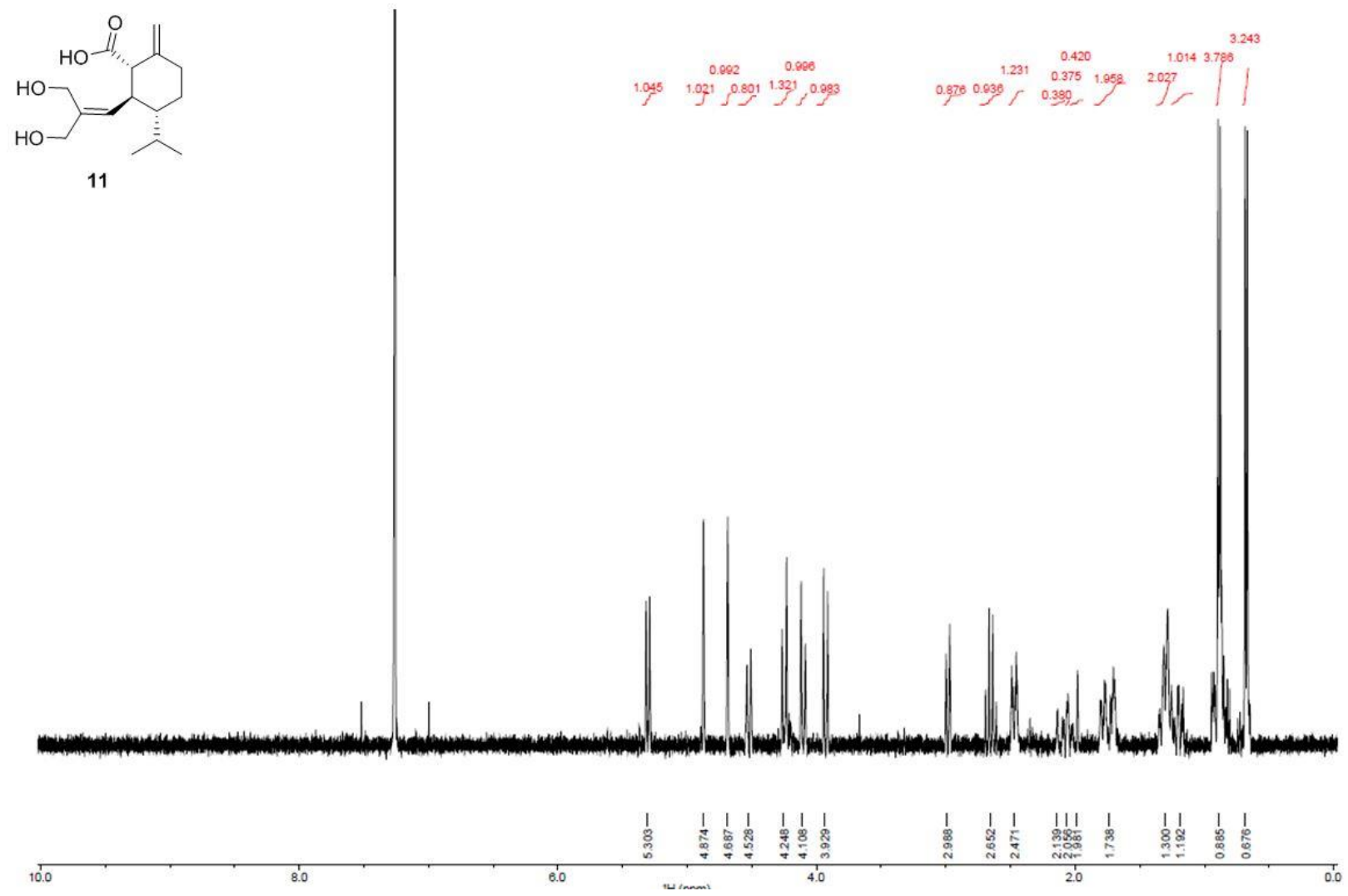


${ }^{1} \mathrm{H} N M R$ spectrum of $12\left(400 \mathrm{MHz}, \mathrm{CDCl}_{3}\right)$

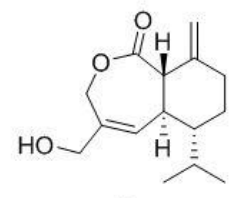

12

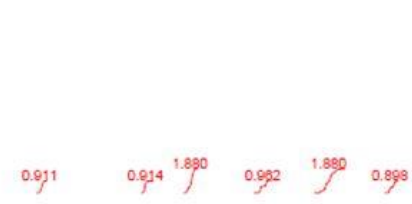

0.8910 .0 .944

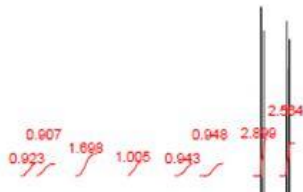

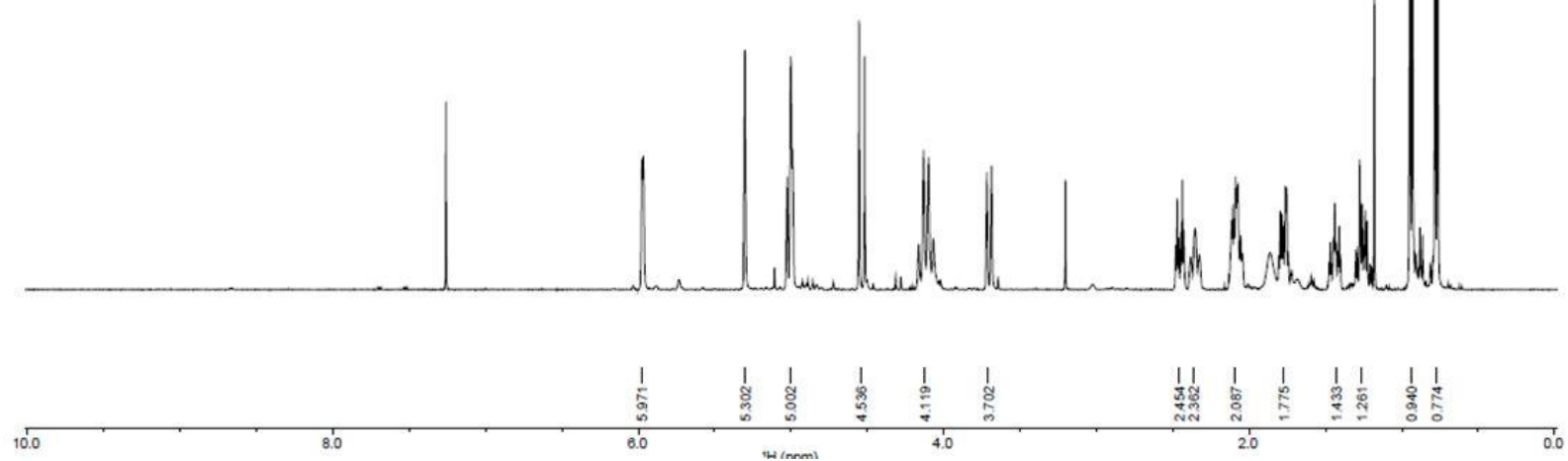


${ }^{1} \mathrm{H} N M R$ spectrum of $13\left(400 \mathrm{MHz}, \mathrm{CDCl}_{3}\right)$

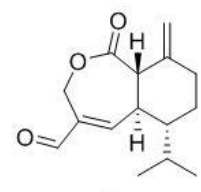

13

1.094

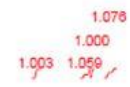

0.900

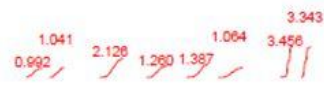

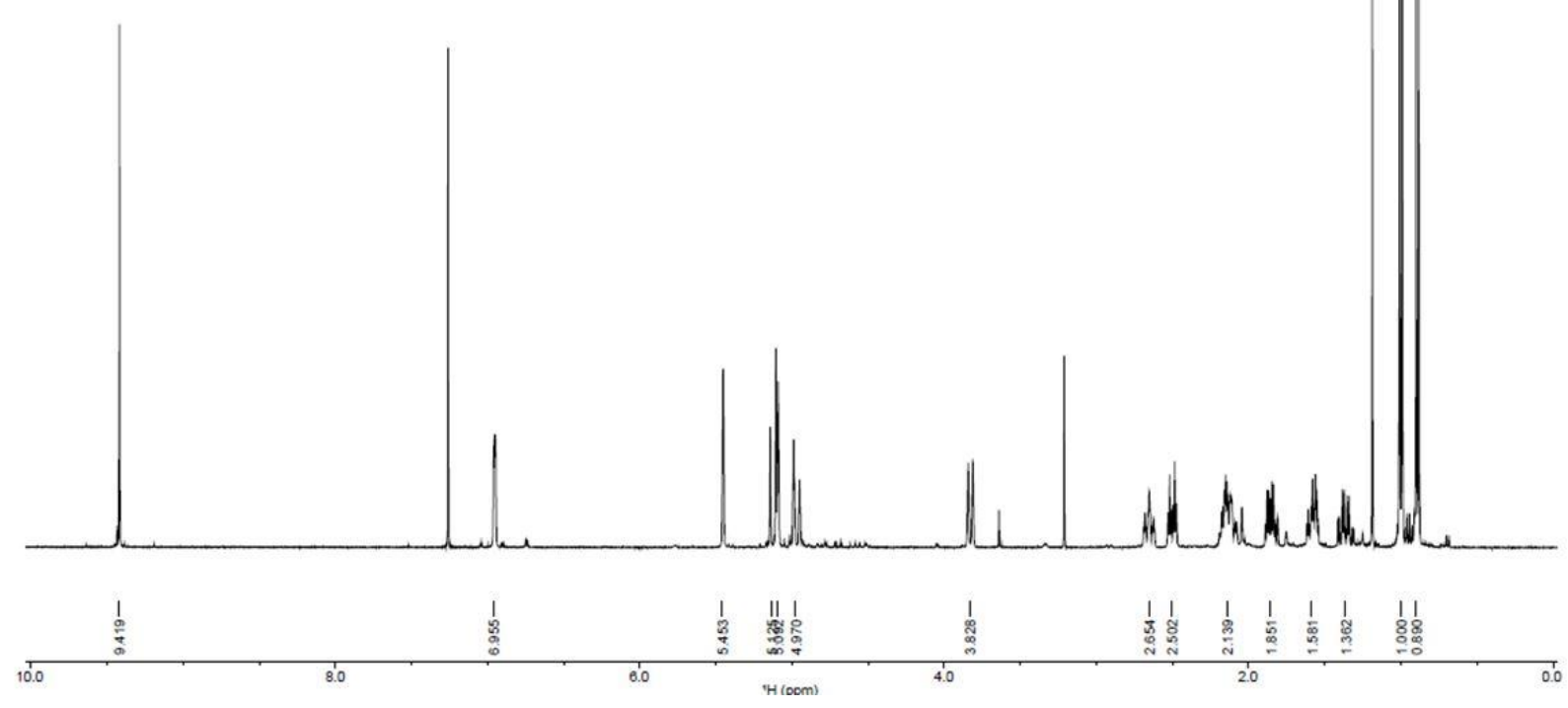


${ }^{1} \mathrm{H} N M R$ spectrum of $14\left(400 \mathrm{MHz}, \mathrm{CDCl}_{3}\right)$

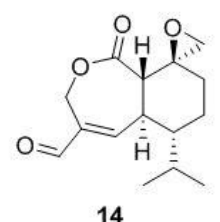

1.021

1.021

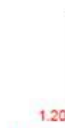

1.193

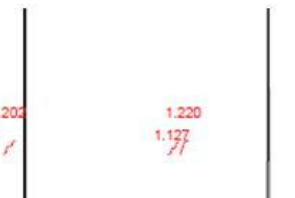

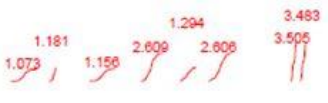

acumblal

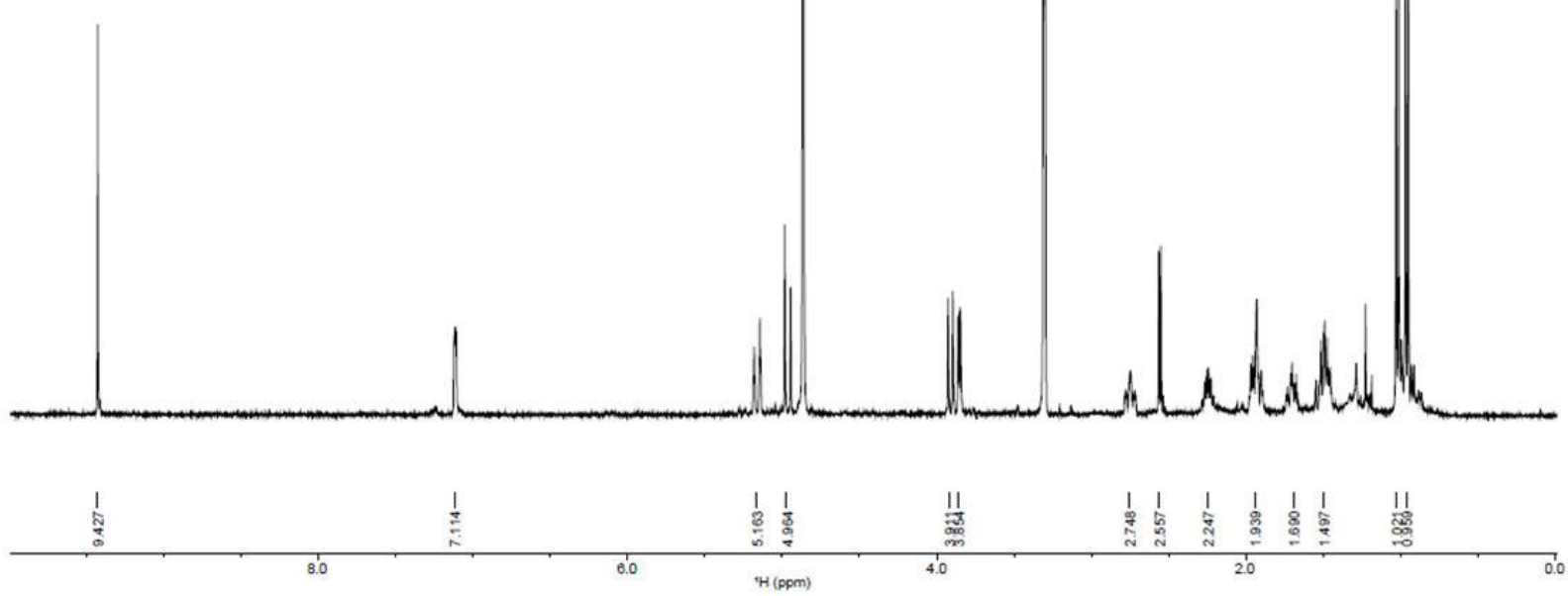


${ }^{l} \mathrm{H} N M R$ spectrum of rac-KA $\left(400 \mathrm{MHz}, \mathrm{MeOH}-d_{4}\right)$<smiles>CC(C)[C@H]1CC[C@H]2O[C@]23C(=O)OCC(C(=O)O)=C[C@H]13</smiles>

Koningic acid

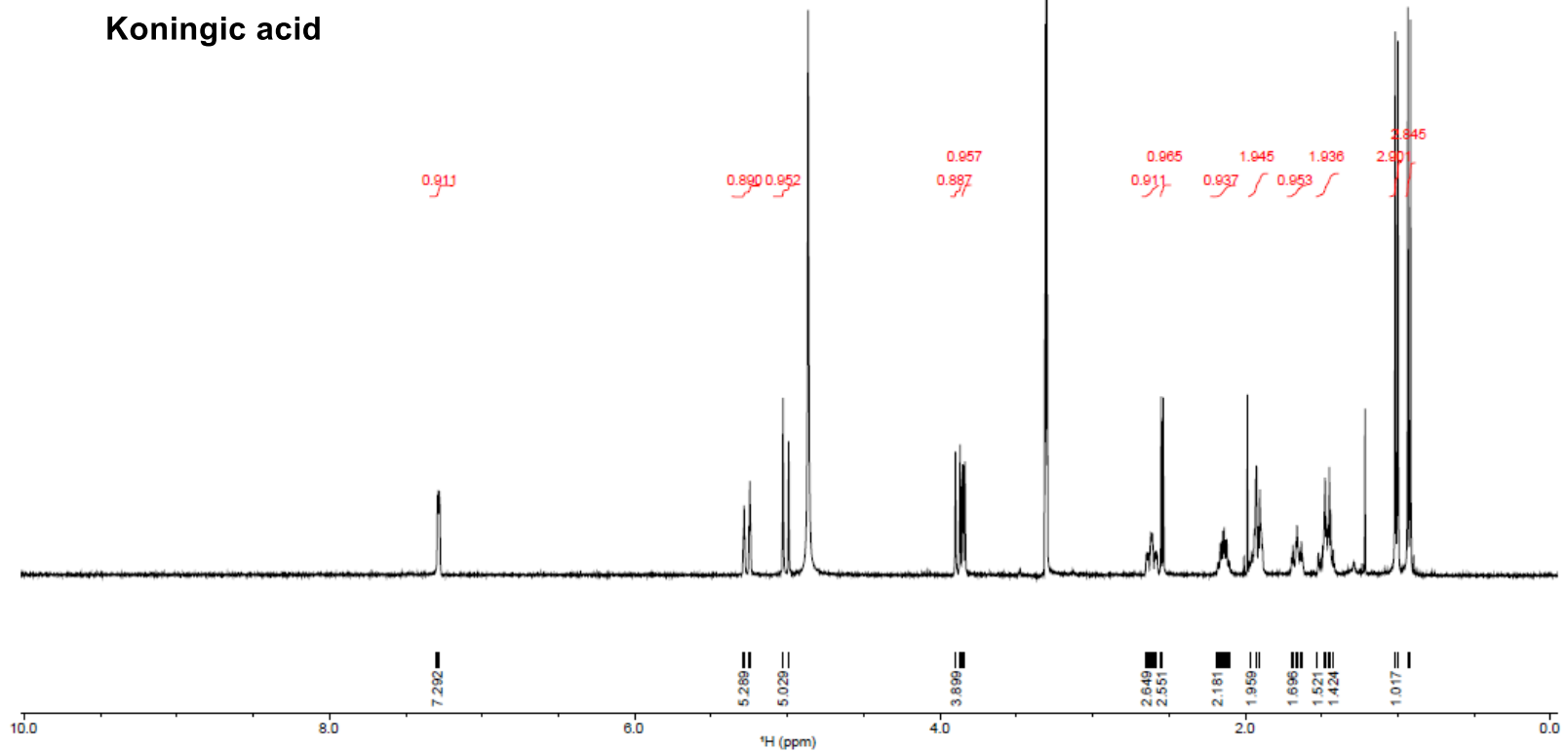

